\title{
Impaired health-related quality of life in adolescents with allergy to staple foods
}

\author{
Jennifer Lisa Penner Protudjer ${ }^{1,2}$, Sven-Arne Jansson ${ }^{1,3}$, Roelinde Middelveld ${ }^{1,2}$, Eva Östblom ${ }^{1,4,5}$,
}

Sven-Erik Dahlén ${ }^{1,2}$, Marianne Heibert Arnlind ${ }^{6,7}$, Ulf Bengtsson ${ }^{8}$, Ingrid Kallström-Bengtsson ${ }^{9}$,

Birgitta Marklund ${ }^{10}$, Georgios Rentzos ${ }^{8}$, Ann-Charlotte Sundqvist ${ }^{4}$, Johanna Åkerström ${ }^{8}$ and Staffan Ahlstedt ${ }^{1,2^{*}}$

\begin{abstract}
Background: Cow's milk, hen's egg and wheat are staple foods in a typical western diet. Despite the ubiquity of these foods, the impact of staple food allergy on health-related quality of life (HRQL) amongst adolescents is incompletely understood. The aims of this study were to make use of the Swedish version of EuroPrevall's disease-specific food allergy quality of life questionnaire-teenager form (FAQLQ-TF) and to investigate the association between objectively-diagnosed staple food allergy and $\mathrm{HRQL}$ amongst adolescents.
\end{abstract}

Methods: In this cross-sectional study, 58 adolescents aged 13-17 years [ $n=40$ (69\%) boys] with objectively-diagnosed allergy to the staple foods cow's milk, hen's egg and/or wheat and living in Stockholm, Sweden were included. Adolescents completed the FAQLQ-TF, which has a corresponding scale of $1=$ best HRQL, and $7=$ worst HRQL. Overall HRQL and domain-specific HRQL were established. Adolescents also reported symptoms, adrenaline auto injector (AAl) prescription and presence of other food allergies. A history of anaphylaxis was defined among those reporting difficulty breathing, inability to stand/collapse, and/or loss of consciousness. Clinically different HRQL was set at a mean difference of $\geq 0.5$.

Results: Overall mean HRQL was poorer than average [mean: 4.70/7.00 (95 \% Cl 4.30-5.01)]. The domain risk of accidental exposure was significantly associated with clinically better HRQL than the domain allergen avoidance and dietary restrictions (mean difference $=0.76 ; p<0.001$ ). Girls had clinically worse, but not statistically significantly different mean HRQL than boys (mean difference $=0.71 ; \mathrm{p}<0.07$ ). HRQL tended to be worse amongst those with allergies to more than three foods or an AAl prescription. The number and types of symptoms, including a history of anaphylaxis were not associated with worse HRQL.

Conclusions: As ascertained via a food allergy-specific questionnaire, adolescents with staple food allergy report poorer than average HRQL, specifically in relation to emerging independence and the need for support. Girls have clinically worse HRQL than boys. The number and type of previous symptoms and history of anaphylaxis were not associated with worse HRQL.

Keywords: Adolescents, Food allergy, Health-related quality of life

\section{Background}

Food allergy affects $2-8 \%$ of adolescents [1, 2]. In this group, health-related quality of life (HRQL) or 'the effects of an illness and its consequent therapy upon a patient, as

\footnotetext{
*Correspondence: staffan.ahlstedt@ki.se

${ }^{1}$ The Centre for Allergy Research, Karolinska Institutet, P.O. Box 287,

17177 Stockholm, Sweden

Full list of author information is available at the end of the article
}

perceived by the patient [3], may be impacted [4-6], particularly in relation to social well-being and independence [7]. Further, adolescents with food allergy report worse overall HRQL compared to matched non-food allergic controls $[4,5]$, or to adolescents with other chronic conditions $[4,8]$. Notably, these studies involved the use of generic HRQL questionnaires $[4,5,8]$, which may not identify the subtleties of food allergy or issues specific to the disease [9]. To address this limitation, EuroPrevall's 
food allergy-specific HRQL questionnaires were specifically developed and validated to glean insights into food allergy that cannot be ascertained by generic questionnaires $[9,10]$. The self-reported adolescent version of this questionnaire, the food allergy quality of life questionnaire-teenager form (FAQLQ-TF), presents a unique means by which to capture perceptions of HRQL of adolescents with food allergy. Self-reported data for this age group is important given the disagreement between adolescent- and parent-reported HRQL [6].

Previous studies on HRQL amongst adolescents with food allergy have focused on a wide range $[4,6]$ or unspecified [5] foods, as well as reported, rather than objectively diagnosed food allergies [5]. However, we believe that objectively diagnosed allergies to certain foods warrant particular attention. For example, allergies to the staple foods cow's milk, hen's egg [11] and wheat [12], typically present in infancy and often exist concomitantly [12]. Although these allergies often resolve by school age [11, 12], those with more severe symptoms or multiple food allergies may experience persistence of staple food allergy through later ages $[11,12]$. As staple foods are ubiquitous in a typical western diet and are consequently difficult to avoid, the HRQL of adolescents experiencing disease persistence is likely to be impacted. Thus, we hypothesised that adolescents with staple food allergies would have poor HRQL, and that adolescents with a history of severe symptoms would have the worst HRQL. To this end, the aims of this study were to make use of the Swedish version of EuroPrevall's FAQLQ-TF and to investigate the association between objectively-diagnosed staple food allergy and HRQL amongst adolescents.

\section{Methods}

\section{Study design and participants}

In this cross-sectional study, adolescents aged 13-17 years with paediatric allergist-diagnosed allergy to one or more staple foods (cow's milk, hen's egg and/ or wheat) were identified from medical records and recruited in 2010-2012 by a paediatric nurse from the outpatient allergy clinic at Sachs' Children and Youth Hospital, Södersjukhuset, in Stockholm, Sweden.

Inclusion criteria were a convincing history of allergy to one or more of the above-mentioned staple foods ascertained either by a positive food challenge with evident symptoms, or by levels of food specific Immunoglobulin $\mathrm{E}$ (IgE) antibodies levels associated with a $95 \%$ probability of a positive result in a double-blind placebo controlled food challenge [13]. Exclusion criteria were an unclear allergy diagnosis to staple food(s), poor understanding of the Swedish language, or presence of coeliac disease, diabetes and/or a malignancy. Information on concomitant allergic disease (asthma, allergic rhinitis, allergic conjunctivitis, eczema) was also obtained. A total of 87 adolescents were eligible and invited to participate. These adolescents were mailed the FAQLQ-TF (described below, English version available as an Additional file 1), as well as an information letter and a postage-paid return envelope. Parents were mailed an information letter and consent form. Completed FAQLQ-TF and signed parental consent forms were received from 58 adolescents (67 \% of those eligible; Fig. 1). Adolescents received two movie tickets following receipt of completed questionnaires. This study was approved by the Regional Ethical Review Board in Stockholm, Sweden (Dnr 2009/84-31/5). Personal data were treated according to the Swedish Personal Data Act.

\section{Exposures}

Both the number of staple food allergies, as well as the number of offending foods (at least one staple food allergy and, participant-reported allergies to other foods) were considered as exposures.

Adolescents responded to 36 closed-ended questions on food allergy symptoms, from which we generated specific symptoms:

Gastrointestinal: stomach upset; vomiting; diarrhoea.

Oral: itchy tongue, mouth or lips; swollen tongue or lips.

Upper respiratory: runny or blocked nose; sneezing.

Lower respiratory: itchy or tight throat; difficulty swallowing; shortness of breath; wheeze; cough.

Cardiovascular/neurological: dizziness; tachycardia; blurred vision; inability to stand/collapse; loss of consciousness.

The most severe symptoms, including difficulty breathing, inability to stand/collapse, and/or loss of consciousness, involved the respiratory- and/or cardiovascular/ neurological systems. In keeping with our previous publications on children [27] and adults [26], and approximating as best as possible the criteria outlined by Sampson et al. [14], such symptoms are collectively referred to as anaphylaxis. Adolescents were asked if they had been prescribed an adrenaline auto injector (AAI).

\section{Outcome \\ Food allergy quality of life questionnaire-teenager form (FAQLQ-TF)}

The FAQLQ-TF [9] was translated into Swedish as per World Health Organization guidelines [15], and was piloted in 10 Swedish-speaking adolescents to ascertain comprehension. Following minor linguistic adjustments, the translation was deemed adequate. The FAQLQ-TF contains 28 questions on HRQL, each of which has corresponding closed-ended answers on a 7-point scale where 1 is best HRQL and 7 is worst HRQL [9]. Overall HRQL established by taking the mean of the 28 questions. These 


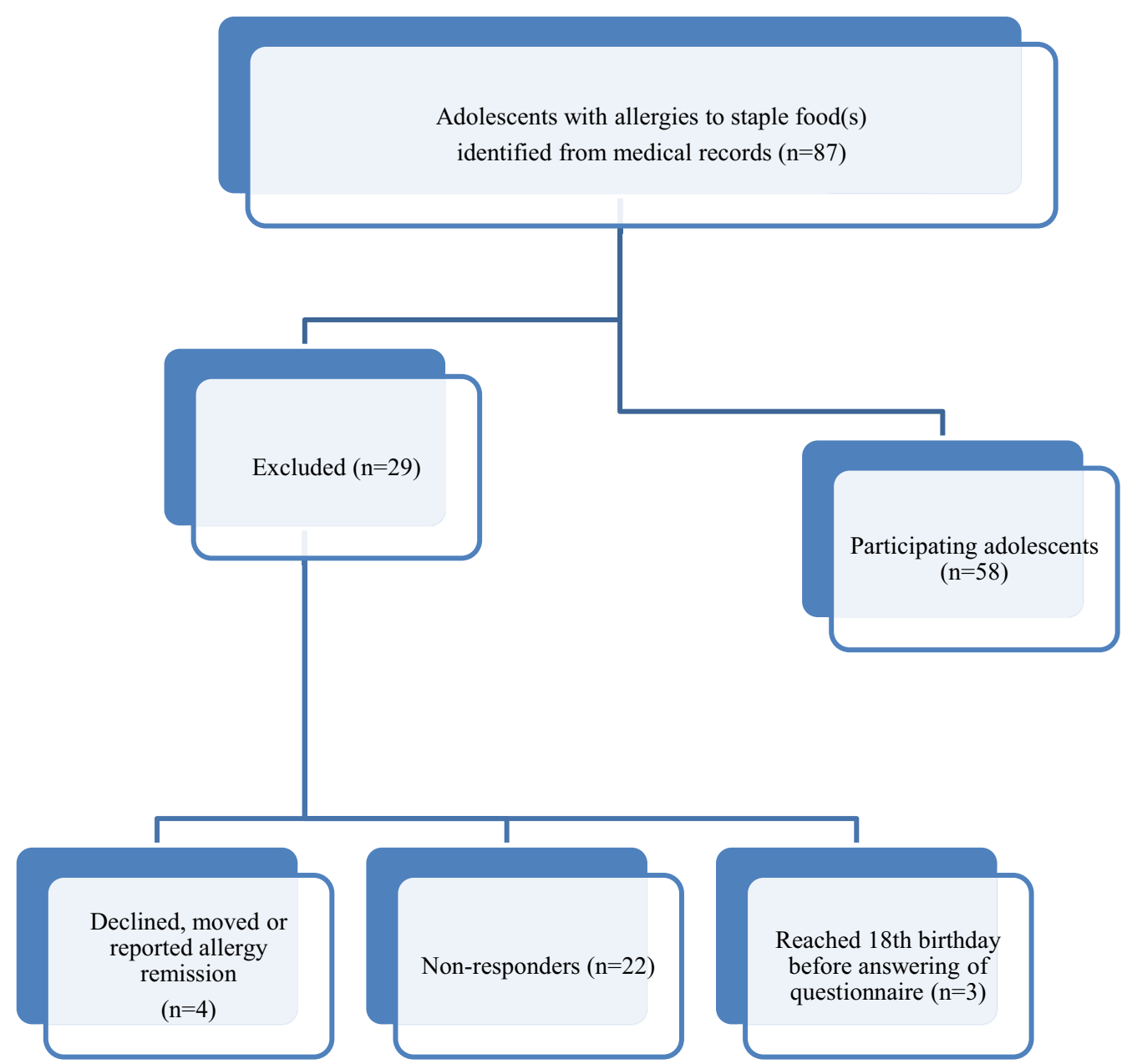

Fig. 1 Flow chart detailing enrolment of adolescents with objectively-diagnosed staple food allergy

questions were also designed to address three domains: allergen avoidance and dietary restrictions (AADR), emotional impact (EI) and risk of accidental exposure (RAE). The first domain, AADR, describes adolescents' perceptions of limitations, hesitations and refusals of foods that they purchase or are offered in social situations. The second domain, EI, reflects adolescents' fears of an allergic reaction or accidental consumption of the food(s) to which they are allergic and their disappointment when others do not take their food allergy seriously. The third domain, RAE, captures adolescents' assessments of needing to be cautious about purchasing food or eating out in relation to changes in ingredients, incorrect disclosure of ingredients and touching certain foods.

\section{Statistics}

Floor and ceiling effects (percentages of patients with minimal and maximum scores, respectively) of the
FAQLQ-TF were calculated to verify discriminative capacity. These effects were considered present if $>15 \%$ of a sample of a minimum of 50 individuals achieved the lowest or highest possible scores, respectively. Absence of these effects demonstrates the efficacy of the questionnaire.

Descriptive statistics included sample sizes (n), percentages, means, parametric two-sample t-tests and $95 \% \mathrm{CI}$. Statistical significance was set at $\mathrm{p}<0.05$. Overall and domain-specific HRQL scores were calculated for the entire study population and stratified by gender. To permit statistical comparisons, the number of staple food allergies was classified into 2 dichotomous groups: 1 vs. $2-3$. The number of offending foods was classified into 4 groups: 1, 2, 3 or $>3$. As described above, adolescents reported on symptoms. Adolescents may forget or inaccurately report their symptoms. Thus, we performed intraclass correlations of adolescent-reported symptoms with 
those reported by their parents as part of a parallel study [32] to measure reliability. These analyses showed modest correlations between adolescent- and parent-reported symptoms, with increasing reliability with increasingly severe symptoms (results not shown). As such, we present the results herein based on adolescent-reported symptoms.

Univariable and multivariable linear regression analyses were performed to identify predictors of HRQL. Potential covariates were identified based on prior knowledge of the exposures and outcome. The covariates gender, number of symptoms, history of anaphylaxis, AAI prescription and concomitant allergic disease were included in the final model as they statistically and independently altered the prediction model. The same models were used for overall and domain-specific HRQL. In keeping with previous publications on HRQL assessed via the FAQLQ, a score of $\geq \pm 0.5$ was considered to be clinically relevant $[9,16]$. Analysis was performed with STATA Statistical Software (release 13.1; StataCorp, College Station, Texas, USA).

\section{Results}

The discriminant capacity of the FAQLQ-TF was confirmed as domain-specific floor and ceiling effects were below $15 \%$ (results not shown).

Our study population included 58 adolescents, of whom 40 (69 \%) were boys (Table 1). Most (62\%) of participants were allergic to only one staple food, although $7 \%$ were allergic to all three staple foods. The most common staple food allergy was to hen's egg (79\%). Other participant-reported allergies to other foods, particularly to tree nuts $(60 \%)$ and peanuts $(53 \%)$, were also common. Nearly all adolescents reported lower respiratory (95\%) and dermatological (90 \%) symptoms. Gastrointestinal symptoms were also common (68\%). Although symptoms involving the cardiovascular/neurological system were the least common, they were nonetheless reported by $27 \%$ of adolescents.

Overall HRQL and domain-specific HRQL are presented in Fig. 2. The overall HRQL mean score was 4.70/7.00 (95 \% CI 4.30-5.01). Girls had clinically worse, but not statistically significantly different HRQL than boys (5.12 \pm 1.01 vs. $4.51 \pm 1.23$, respectively; mean difference $=0.71 ; \mathrm{p}<0.07)$.

With consideration to the different domains, the mean scores for AADR and EI were comparable (Fig. 2). In contrast, RAE was significantly associated with clinically better HRQL compared to the reference domain, AADR (mean 4.19/7.00; 95 \% CI 3.82-4.56 vs. mean 4.95/7.00; $95 \%$ CI 4.65-5.25, respectively; mean difference $=0.76$; $\mathrm{p}<0.001$ ). Only the domain, EI, differed significantly between the sexes, with clinically worse HRQL amongst
Table 1 Descriptive allergy characteristics of adolescents with objectively-diagnosed staple food allergy

\begin{tabular}{|c|c|c|}
\hline & $\mathbf{n}$ & $\%$ \\
\hline \multicolumn{3}{|l|}{ Sex } \\
\hline Boys & 40 & 69.0 \\
\hline Girls & 18 & 31.0 \\
\hline \multicolumn{3}{|c|}{ Number of staple food allergies } \\
\hline 1 & 36 & 62.1 \\
\hline 2 & 18 & 31.0 \\
\hline 3 & 4 & 6.9 \\
\hline \multicolumn{3}{|c|}{ Offending staple foods ${ }^{\mathrm{a}}$} \\
\hline Hen's egg & 46 & 79.3 \\
\hline Cow's milk & 29 & 50.0 \\
\hline Wheat & 5 & 8.6 \\
\hline \multicolumn{3}{|c|}{ Participant-reported allergies to other foods ${ }^{\mathrm{a}}$} \\
\hline Tree nuts & 35 & 60.3 \\
\hline Peanuts & 31 & 53.4 \\
\hline Fruit & 17 & 29.3 \\
\hline Vegetables & 8 & 13.8 \\
\hline Fish & 9 & 15.5 \\
\hline Shellfish & 12 & 20.7 \\
\hline Soy & 6 & 10.3 \\
\hline Sesame seeds & 2 & 3.4 \\
\hline \multicolumn{3}{|c|}{ Number of offending foods ${ }^{b}$} \\
\hline 1 & 8 & 13.8 \\
\hline 2 & 10 & 17.2 \\
\hline 3 & 13 & 22.4 \\
\hline$>3$ & 27 & 46.6 \\
\hline \multicolumn{3}{|c|}{ Symptoms resulting from staple foods ${ }^{a}$} \\
\hline Gastrointestinal & 28 & 68.3 \\
\hline Dermatological & 37 & 90.2 \\
\hline Oral cavity & 32 & 39.0 \\
\hline Upper respiratory & 16 & 39.0 \\
\hline Lower respiratory & 39 & 95.1 \\
\hline Cardiovascular & 11 & 26.8 \\
\hline Anaphylaxis & 23 & 56.1 \\
\hline \multicolumn{3}{|c|}{ Concomitant allergic disease ${ }^{c}$} \\
\hline None or one & 11 & 19.0 \\
\hline Two or more & 47 & 81.0 \\
\hline
\end{tabular}

${ }^{a}$ Not mutually exclusive

b Includes at least 1 objectively-diagnosed staple food, as well as any participant-reported allergies to other foods

c Asthma, allergic rhinitis, allergic conjunctivitis and/or eczema

girls than boys $(5.38 \pm 1.4$ vs. $4.50 \pm 0.24$; mean difference $=0.88 ; \mathrm{p}<0.04)$.

Investigation to the individual questions of each domain revealed further insights into the fine tuning of the HRQL of the different domains. For example, in the domain AADR, compared to the question with the highest mean score (i.e. worst HRQL), 'How troublesome 


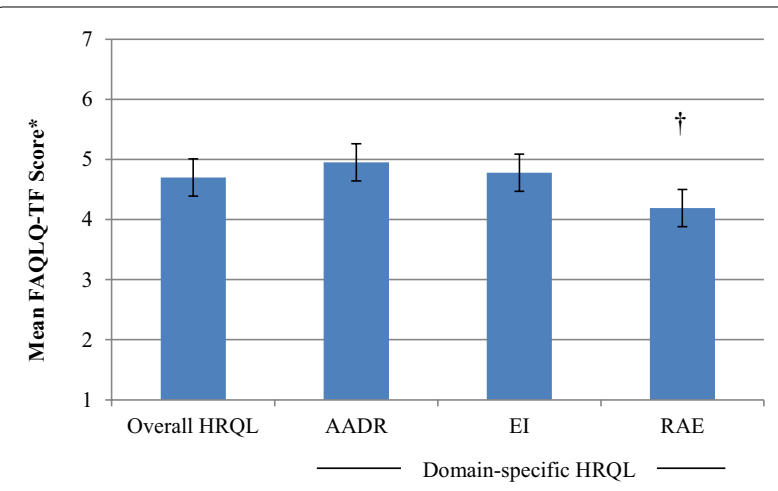

Fig. 2 Overall- and domain-specific HRQL mean scores and $95 \% \mathrm{Cl}$ for adolescents with objectively-diagnosed staple food allergy. Asterisk FAQLQ-TF on a scale of 1-7, where 1 corresponds to best HRQL and 7 corresponds to worst HRQL; based on self-report. † Compared to AADR $(p<0.001)$. HRQL health-related quality of life, AADR allergen avoidance and dietary restrictions, El emotional impact, RAE risk of accidental exposure

do you find it, because of your food allergy, that you must check yourself whether you can eat something when eating out', factors that were associated with significantly better HRQL included limitations on eating, buying or refusing foods, or spontaneously accepting invitations to a meal. Within the domain, EI, compared to the reference question, 'How disappointed are you when people don't take your food allergy into account?', factors associated with significantly better HRQL included feeling discouraged, carrying an AAI, or fears related to eating something 'wrong' or something new. Within the domain, RAE, compared to the reference question, 'How troublesome do you find it, because of your food allergy, that you have to explain to people around you that you have a food allergy?', the only factor associated with significantly better HRQL related to labelling discrepancies between bulk and individual packaging (Table 2).

In linear regression analyses adjusted for sex, number of symptoms, history of anaphylaxis, AAI prescription and concomitant allergic disease (excluding the predictor), girls had clinically worse and but not statistically significant HRQL than boys $(B=-0.58 ; 95 \% \mathrm{CI}-1.34$; $0.19 ; \mathrm{p}=0.13$; Table 3). Similarly, allergies to $>3$ staple food allergies and AAI prescription reached the threshold of $\geq 0.5$ for clinical relevance but only trended towards significance ( $\mathrm{p}=0.13$ and $\mathrm{p}=0.06$, respectively).

Consideration to the presence vs. absence of specific symptoms including anaphylaxis attributable to staple foods yielded no statistically significant associations. Only previous lower respiratory symptoms reached the threshold for clinically better HRQL $(B=0.67 ; 95 \%$ CI $-1.37 ; 2.70, \mathrm{p}=0.51$; Table 4).

\section{Discussion}

In this cross-sectional study of adolescents with objectively-diagnosed staple food allergies to cow's milk, hen's egg and/or wheat, overall HRQL, as ascertained by a food allergy specific questionnaire, was poorer than average. The domain risk of accidental exposure was significantly associated with clinically better HRQL than the domain allergen avoidance and dietary restrictions. Girls had clinically worse, but not statistically significantly different HRQL than boys. HRQL tended to be worse amongst adolescents with allergies to more than three foods or those who had been prescribed an AAI. In contrast, the number and type of previous symptoms and history of anaphylaxis were not associated with worse HRQL.

We highlight the key strengths of this study we used a food allergy-specific questionnaire that is robust for adolescents with food allergies $[9,17]$ that provided insights into associations between food allergy and HRQL that could not have been gleaned via a generic questionnaire [9]. Similarly, this work presents the first results of the Swedish version of the FAQLQ-TF. In our study, participants had objectively-diagnosed allergies, thus providing insight into the impact of true, rather than perceived, food allergy on HRQL. Amongst children, HRQL does not differ between these two phenotypes [18]. However, consideration of the association between objectivelydiagnosed allergy and adolescent-reported HRQL warrants consideration as this age group is increasingly responsible for their own food choices, and thus the potential consequences of inappropriate food choices. Although adolescents' food choices are influenced by their allergies, they also make choices based on peers and sensory preferences [19]. We believe that we are the first group to report on HRQL amongst adolescents with allergies to foods that are ubiquitous in a typical western diet, but which also receive less attention in relation to HRQL than other common food allergens, such as peanuts or tree nuts.

We also acknowledge the limitations of our study. Our study was cross-sectional in design, thereby precluding establishment of a causal relationship between staple food allergy and HRQL. As well, our study population included more boys than girls. As evidenced in the general adolescent population [20], and amongst food hypersensitive adolescents for whom HRQL was established using a generic questionnaire [5], boys generally have better HRQL than girls. Thus, we surmise that, had adolescent boys and girls been equally represented, overall HRQL may have been even worse.

The FAQLQ-TF is specifically designed to capture adolescents' perceptions of HRQL [9]. Elsewhere, responses from Dutch adolescent-parent pairs to the FAQLQ have been compared [6]. In that study, adolescents were 
Table 2 Mean scores for individual questions used to calculate domain-specific HRQL

\begin{tabular}{|c|c|c|}
\hline & Mean & $p$ value $^{t}$ \\
\hline \multicolumn{3}{|l|}{ Allergen avoidance and dietary restrictions } \\
\hline \multicolumn{3}{|l|}{ How troublesome do you find it, because of your food allergy, that you: } \\
\hline Must check yourself whether you can eat something when eating out? & 5.76 & \\
\hline Must read labels? & 5.74 & 0.95 \\
\hline Hesitate eating a product when you have doubts about it? & 5.67 & 0.75 \\
\hline Must always be alert to what you are eating? & 5.66 & 0.70 \\
\hline Are less able to taste or try various products when eating out? & 5.36 & 0.15 \\
\hline Are able to eat fewer products? & 5.21 & $<0.05$ \\
\hline Are limited as to the products that you can buy? & 4.47 & $<0.001$ \\
\hline Are less able to spontaneously accept an invitation to stay for a meal? & 4.09 & $<0.001$ \\
\hline Must be careful about touching certain foods? & 3.47 & $<0.001$ \\
\hline Must refuse treats at school or work? & 2.58 & $<0.001$ \\
\hline \multicolumn{3}{|l|}{ Emotional impact } \\
\hline \multicolumn{3}{|l|}{ Answer the following: } \\
\hline How disappointed are you when people don't take your food allergy into account? & 5.50 & \\
\hline How discouraged do you feel during an allergic reaction? & 4.74 & $<0.05$ \\
\hline \multicolumn{3}{|l|}{ How troublesome do you find it, because of your food allergy, that you: } \\
\hline Have the feeling that you have less control of what you eat when eating out? & 5.40 & 0.86 \\
\hline Must carry an EpiPen ${ }^{\circledR}$ ? & 4.36 & $<0.01$ \\
\hline \multicolumn{3}{|l|}{ How frightened are you because of your food allergy: } \\
\hline Of accidentally eating something wrong? & 4.59 & $<0.05$ \\
\hline Of an allergic reaction? & 4.53 & $<0.05$ \\
\hline To eat something that you have never eaten before? & 4.31 & $<0.05$ \\
\hline \multicolumn{3}{|l|}{ Risk of accidental exposure } \\
\hline \multicolumn{3}{|l|}{ How troublesome do you find it, because of your food allergy, that you: } \\
\hline That you have to explain to people around you that you have a food allergy? & 5.00 & \\
\hline That during social activities others can eat the food to which you are allergic? & 4.95 & 0.88 \\
\hline That during social activities your food allergy is not taken into account enough? & 4.47 & 0.17 \\
\hline That the ingredients of a food change? & 4.45 & 0.10 \\
\hline That the label states: "May contain (traces of)...."? & 4.43 & 0.15 \\
\hline That the labelling of the bulk packaging (e.g. box or bag) is different than the individual packages? & 3.27 & $<0.001$ \\
\hline
\end{tabular}

FAQLQ-TF on a scale of 1-7, where 1 corresponds to best HRQL and 7 corresponds to worst HRQL; based on self-report

${ }^{\dagger}$ Compared to the individual question with the highest mean score (i.e. worst HRQL) within each domain

allergic to a wide range of common food allergens, but staple food allergy did not predominate. Amongst these adolescent-parent pairs, adolescents reported clinically worse, but not statistically significant differences in HRQL. This finding underscores the need to specifically query adolescents' perceptions of HRQL and address not only statistical differences but consideration to the magnitude of the differences. Interestingly, our domainspecific scores exceeded the clinically relevant difference of $\geq 0.5$ compared to the Dutch study [6], as well as other studies in which the FAQLQ-TF was used [6, $9,17,21]$. Collectively, these studies suggest that staple food allergy is associated with worse HRQL than allergies to other foods. One can speculate that this may be due to the ubiquity of staple foods in a typical western diet, making them challenging to avoid. This challenge may be compounded by the fact that, although many countries have regulatory frameworks for allergens contained in processed foods [22], such labelling most commonly identifies non-staple foods [23] and may contribute to confusion and complacency amongst food allergic individuals $[24,25]$.

We identified that staple food allergy impacted on adolescents' lifestyles, as underscored by poorer than average overall HRQL mean score [i.e. better HRQL], as well as within the domain, AADR. Others have reported similar findings on the impact of food allergy on adolescents' lifestyles [6]. The similarities between these findings are 
Table 3 Linear regression analyses of HRQL for adolescents with objectively-diagnosed staple food allergy

\begin{tabular}{|c|c|c|c|c|}
\hline & $\mathbf{n}$ & $B$ & $95 \% \mathrm{Cl}$ for $B$ & $\mathrm{p}$ value \\
\hline \multicolumn{5}{|l|}{ Sex } \\
\hline Boys & 18 & Ref & & \\
\hline Girls & 40 & -0.58 & $-1.34 ; 0.19$ & 0.13 \\
\hline \multicolumn{5}{|c|}{ Number of staple food allergies } \\
\hline 1 & 36 & Ref & & \\
\hline $2-3$ & 22 & 0.37 & $-0.31 ; 1.06$ & 0.28 \\
\hline \multicolumn{5}{|c|}{ Number of offending foods ${ }^{\mathrm{a}}$} \\
\hline 1 & 10 & Ref & & \\
\hline 2 & 9 & 0.79 & $-0.43 ; 2.01$ & 0.20 \\
\hline 3 & 13 & 0.03 & $-1.11 ; 1.17$ & 0.95 \\
\hline$>3$ & 26 & 0.82 & $-0.24 ; 1.89$ & 0.13 \\
\hline \multicolumn{5}{|c|}{ Number of symptoms resulting from staple foods } \\
\hline $0-3$ & 15 & Ref & & \\
\hline $4-6$ & 26 & 0.10 & $-0.87 ; 1.07$ & 0.83 \\
\hline \multicolumn{5}{|c|}{ Adrenaline auto injector possession } \\
\hline No & 14 & Ref & & \\
\hline Yes & 44 & 0.78 & $-0.02 ; 1.57$ & 0.06 \\
\hline
\end{tabular}

Adjusted for the covariates: sex, number of symptoms, history of anaphylaxis, adrenaline auto injector prescription and concomitant allergic disease, excluding predictor

a Includes at least 1 objectively-diagnosed staple food, as well as participantreported allergies to other foods

not surprising, as adolescents spend increasing amounts of time in social settings away from home. However, our findings extend those of previous studies, as we were able to disentangle the specific factors that contributed most to worse HRQL.

In the present study, adolescents reported worse HRQL related to emerging independence and the need for support, as evidenced by domain-specific mean scores which were highest for the questions relating to needing to check for themselves if they can eat a food whilst dining out, expressing disappointment when their food allergies are not taken into account, or explaining to others about their food allergy. Previously, we reported that differences between the domains were present amongst adults [26], but not children [27] with staple food allergy. As with adults [26] and children [27], adolescents with staple food allergy reported significantly worse HRQL if they had multiple food allergies or possessed an AAI. Likewise, both adolescents and adults [26] reported worse HRQL within the domain AADR. As different domains were defined for children, a comparison of the domains between children and adolescents is not possible. In contrast to both adults [26] or children [27], adolescents did not report worse HRQL in association with a history of anaphylaxis or the number and type of symptoms. Taken together, it could be
Table 4 Linear regression analyses for presence vs. absence of specific symptoms resulting from staple foods in adolescents with objectively-diagnosed staple food allergy

\begin{tabular}{|c|c|c|c|c|}
\hline & $\mathrm{n}$ & $B$ & $95 \% \mathrm{Cl}$ for $B$ & $p$ value \\
\hline \multicolumn{5}{|c|}{ Gastrointestinal } \\
\hline No & 13 & Ref & & \\
\hline Yes & 28 & 0.02 & $-1.04 ; 1.08$ & 0.97 \\
\hline \multicolumn{5}{|c|}{ Dermatological ${ }^{\mathrm{a}}$} \\
\hline No & 4 & Ref & & \\
\hline Yes & 37 & 0.41 & $-1.86 ; 2.68$ & 0.72 \\
\hline \multicolumn{5}{|l|}{ Oral } \\
\hline No & 9 & Ref & & \\
\hline Yes & 32 & 0.41 & $-0.98 ; 1.80$ & 0.57 \\
\hline \multicolumn{5}{|c|}{ Upper respiratory } \\
\hline No & 25 & Ref & & \\
\hline Yes & 16 & 0.29 & $-0.88 ; 1.44$ & 0.62 \\
\hline \multicolumn{5}{|c|}{ Lower respiratory $^{\mathrm{a}}$} \\
\hline No & 2 & Ref & & \\
\hline Yes & 39 & 0.67 & $-1.37 ; 2.70$ & 0.51 \\
\hline \multicolumn{5}{|c|}{ Cardiovascular } \\
\hline No & 30 & Ref & & \\
\hline Yes & 11 & 0.29 & $-0.88 ; 1.45$ & 0.62 \\
\hline \multicolumn{5}{|c|}{ History of anaphylaxis } \\
\hline No & 18 & Ref & & \\
\hline Yes & 23 & -0.33 & $-1.23 ; 0.58$ & 0.46 \\
\hline
\end{tabular}

Adjusted for the covariates: sex, number of symptoms, history of anaphylaxis, adrenaline auto injector prescription and concomitant allergic disease, excluding predictor

Non-mutually exclusive symptoms

a Interpret with caution due to small counts

speculated that children may not feel burdened by the dietary restrictions imposed by staple food allergy, likely as the responsibility for safe food choices is assumed by their parents/guardians, whereas adolescents and adults do have to assume this responsibility. The burden of dietary restrictions and safe food choices has also been qualitatively explored amongst adolescents. Unlike nonfood allergic adolescents, those with food allergies feel safe under parental control and thus do not necessarily want to make food-related decisions independently [19]. Adolescents with food allergy describe themselves as being very mature for their age, yet dependent on others in the event of a reaction [28]. One can speculate that these opposing characteristics may result in worse food allergy-specific HRQL.

Unlike other chronic conditions which adolescents may neglect or be non-compliant [29], food allergy cannot be ignored for longer than an interval between meals or snacks. But given that food is an integral part of social events, feelings of exclusion and 'being different' may 
ensue [7]. This may begin to explain why food allergy is strongly associated with worse HRQL. This may also explain why neither the number of allergies or symptoms, nor AAI prescription is a predictor of clinically worse HRQL. Further, adolescents often base their food choices primarily on enjoyment and secondarily on content [19], thereby engaging in risk taking behaviours [30] that may potentially lead to severe reactions. Yet, like others [9], we found no difference in HRQL between those with vs. without a history of anaphylaxis.

The financial burden of food allergy on healthcare systems [31] and on households [32] is high, and allergyrelated hospitalisations are increasing [33]. Worse HRQL also predicts greater healthcare costs [34]. Thus, addressing HRQL amongst adolescents, as well as children [27] and adults [26] with staple food allergy warrants considerable attention.

\section{Conclusions}

As ascertained via a food allergy-specific questionnaire, adolescents with staple food allergy report poorer than average $\mathrm{HRQL}$, specifically in relation to emerging independence and the need for support. Girls have clinically worse HRQL than boys. The number and type of previous symptoms and history of anaphylaxis were not associated with worse HRQL (Additional file 1.).

\section{Additional file}

Additional file 1. Food allergy quality of life questionnaire-teenager form (13-17 years).

\section{Abbreviations}

AADR: allergen avoidance and dietary restrictions; AAl: adrenaline auto-injector; El: emotional impact; FAQLQ-TF: food allergy quality of life questionnaireteenager form; HRQL: health-related quality of life; RAE: risk of accidental exposure; $95 \% \mathrm{Cl}$ : 95 th percent confidence interval.

\section{Authors' contributions}

JP contributed to the design of this manuscript, performed the data analyses and formulated the draft manuscripts. SAJ contributed to the study design, and provided critical comments on the results as well as on the draft manuscripts. RM contributed to the study design, scrutinized the questionnaires and letters, contributed to the interpretation of the data analyses and provided critical comments on the draft manuscripts. EÖ contributed to the study design and collection of the patients, and provided critical comments on the draft manuscripts. SED contributed to the study design, was instrumental for the grant applications for the study, scrutinized the results and their interpretation and critiqued the draft manuscripts. MHA contributed to the study design, formulation of the questionnaires and provided comments on the draft manuscripts. UB contributed to the study design, formulation of the questionnaires and provided comments on the draft manuscripts. IK-B contributed to the study design, formulation of the questionnaires and provided comments on the draft manuscripts. BM contributed to the study design, formulation of the questionnaires and provided comments on the draft manuscripts. GR contributed to the study design, formulation of the questionnaires and provided comments on the draft manuscripts. A-CS contributed to the selection of the patients and their diagnosis, formulation of the questionnaires as well as interpretation of the analysis of the results. JÅ contributed to the formulation of the questionnaires as well as interpretation of the analysis of the results. SA headed the project, contributed to the study design, contributed to the formulation of the questionnaires, contributed to the interpretation and presentation of the results and provided critical comments on the draft manuscripts. All authors read and approved the final manuscript.

\section{Author details}

${ }^{1}$ The Centre for Allergy Research, Karolinska Institutet, P.O. Box 287, 17177 Stockholm, Sweden. ${ }^{2}$ Institute of Environmental Medicine, Karolinska Institutet, Stockholm, Sweden. ${ }^{3}$ Department of Public Health and Clinical Medicine, Umeå University, Umeå, Sweden. ${ }^{4}$ Sachs' Children and Youth Hospital, Södersjukhuset, Stockholm, Sweden. ${ }^{5}$ Department of Clinical Research and Education, Södersjukhuset, Karolinska Institutet, Stockholm, Sweden.

${ }^{6}$ Swedish Council on Health Technology Assessment, SBU, Stockholm,

Sweden. ${ }^{7}$ Department of Learning, Informatics, Management and Ethics, and Medical Management Centre, Karolinska Institutet, Stockholm, Sweden.

${ }^{8}$ Allergy Unit, Sahlgrenska University Hospital, Gothenburg, Sweden. ${ }^{9}$ The

Swedish Asthma and Allergy Foundation, Stockholm, Sweden. ${ }^{10}$ Department of Health and Caring Sciences, Linnaeus University, Kalmar, Sweden.

\section{Acknowledgements}

We acknowledge Ms. Margareta Andersson for help with organising questionnaire postage and data entry.

\section{Competing interests}

The authors declare that they have no competing interests.

\section{Availability of data and materials}

An English version of the FAQLQ-TF is available as an online supplement associated with this publication.

Due to the relatively low number of cases $(n=58)$ and the strict inclusion criteria (objectively-diagnosed allergy to one or more staple foods), we believe that cases may potentially be identifiable if online data were to be included.

\section{Consent for publication}

All authors have approved the final version of the manuscript prior to publication

\section{Ethics approval and consent to participate}

This study was approved by the Regional Ethical Review Board in Stockholm, Sweden (Dnr 2009/84-31/5). Personal data were treated according to the Swedish Personal Data Act.

Parents provided written consent prior to completing the questionnaires.

\section{Funding}

This study was funded by The Centre for Allergy Research (CfA) at the Karolinska Institutet, the EU project EuroPrevall (FP6 contract number FOODCT-2005-514000), the Swedish Asthma and Allergy Foundation and the Sahlgrenska University Hospital. Jennifer LP Protudjer is a postdoctoral researcher funded by the Karin and Sten Mörtstedt Initiative on Anaphylaxis and the Swedish Allergy and Asthma Foundation.

Received: 5 April 2016 Accepted: 19 September 2016

Published online: 30 September 2016

\section{References}

1. Rona RJ, Keil T, Summers C, Gislason D, Zuidmeer L, Sodergren E, et al. The prevalence of food allergy: a meta-analysis. J Allergy Clin Immunol. 2007;120(3):638-46.

2. Sicherer SH, Sampson HA. Food allergy: epidemiology, pathogenesis, diagnosis, and treatment. J Allergy Clin Immunol. 2014;133(2):291-307.

3. de Blok BMJ, Dubois AE, Hourihane JO. The impact of food allergy on quality of life. In: Mills ENC, Wichers H, Hoffmann-Sommergruber K, editors. Managing allergens in food. Cambridge: Woodhead Publishing; 2006.

4. Flokstra-de Blok BM, Dubois AE, Vlieg-Boerstra BJ, Oude Elberink JN, Raat H, DunnGalvin A, et al. Health-related quality of life of food allergic patients: comparison with the general population and other diseases. Allergy. 2010;65(2):238-44. 
5. Marklund B, Ahlstedt S, Nordström G. Health-related quality of life among adolescents with allergy-like conditions - with emphasis on food hypersensitivity. Health Qual Life Outcomes. 2004;2:65.

6. van der Velde JL, Flokstra-de Blok BM, Hamp A, Knibb RC, Duiverman EJ, Dubois AE. Adolescent-parent disagreement on health-related quality of life of food-allergic adolescents: who makes the difference? Allergy. 2011;66(12):1580-9.

7. DunnGalvin A, Dubois AE, Flokstra-de Blok BM, Hourihane JO. The effects of food allergy on quality of life. Chem Immunol Allergy. 2015;101:235-52.

8. Mo F, Choi BC, Li FC, Merrick J. Using health utility index (HUI) for measuring the impact on health-related quality of life (HRQL) among individuals with chronic diseases. Sci World J. 2004;4:746-57.

9. Flokstra-de Blok BM, DunnGalvin A, Vlieg-Boestra BJ, Oude Elbernik JN, Duiverman EJ, Hourihane JO, et al. Development and validation of the self-administered food allergy quality of life questionnaire for adolescents. J Allergy Clin Immunol. 2008;122(1):139-44.

10. Salvilla SA, Dubois AEJ, Flokstra-de Blok BM, Panesar SS, Worth A, Patel S, et al. Disease-specific health-related quality of life instruments for lgEmediated food allergy. Allergy. 2014;69:834-44.

11. Burks AW, Land MH. Long-term follow-up of IgE-mediated food allergy: determining persistence versus clinical tolerance. Ann Allergy Asthma Immunol. 2014;112:200-6.

12. Keet CA, Matsui EC, Dhillon G, Lenehan P, Paterakis M, Wood RA. The natural history of wheat allergy. Ann Allergy Asthma Immunol. 2009;2009(102):5.

13. Boyce JA, Assa'ad A, Burks AW, Jones SM, Sampson HA, Wood RA, et al. Guidelines for the diagnosis and management of food allergy in the United States: report of the NIAID-sponsored expert panel. J Allergy Clin Immunol. 2010;126(6 Suppl):S1-58.

14. Sampson HA, Muñoz-Furlong A, Campbell RL, Adkinson NF Jr, Bock SA, Branum A, et al. Second symposium on the definition and management of anaphylaxis: summary report-second National Institute of Allergy and Infectious Disease/Food Allergy and Anaphylaxis Network symposium. Ann Emerg Med. 2006;47(4):373-80.

15. World Health Organization. Process of translation and adaptation of instruments. 2013. http://www.who.int/substance_abuse/research_tools/ translation/en/. Accessed 4 Nov 2013.

16. Saleh-Langenberg J, Goossens NJ, Flokstra-de Blok BM, Kollen BJ, van der Meulen GN, Le TM, et al. Predictors of health-related quality of life of European food-allergic patients. Allergy. 2015;70(6):616-24.

17. van der Velde JL, Flokstra-de Blok BM, Vlieg-Boerstra BJ, Oude Elberink JNG, Schouten JP, DunnGalvin A, et al. Test-retest reliability of the food allergy quality of life questionnaires (FAQLQ) for children, adolescents and adults. Qual Life Res. 2009;18(2):245-51.

18. Venter C, Sommer I, Moonesinghe H, Grundy J, Glasbey G, Dean T. Healthrelated quality of life in children with perceived and diagnosed food hypersensitivity. Pediatr Allergy Immunol. 2015;26(2):126-32.

19. Sommer I, Mackenzie H, Venter C, Dean T. An exploratory investigation of food choice behavior of teenagers with and without food allergies. Ann Allergy Asthma Immunol. 2014;112(5):446-52.
20. Group K, Michel G, Bisegger C, Fuhr DC, Abel T. Age and gender differences in health-related quality of life of children and adolescents in Europe: a multilevel analysis. Qual Life Res. 2009;18(9):1147-57.

21. Flokstra-de Blok BM, van der Velde JL, Vlieg-Boerstra BJ, Oude Elberink JNG, DunnGalvin A, Hourihane JO, et al. Health-related quality of life of food allergic patients measured with generic and disease-specific questionnaires. Allergy. 2010;65(8):1031-8.

22. Taylor SL, Baumert JL. Worldwide food allergy labeling and detection of allergens in processed foods. Chem Immunol Allergy. 2015;101:27-34.

23. Zurzolo GA, Mathai ML, Koplin JJ, Allen KJ. Precautionary allergen labelling following new labelling practice in Australia. J Paediatr Child Health. 2013;49(4):E306-10

24. Barnett J, Muncer K, Leftwich J, Shepherd R, Raats MM, Gowland MH, et al. Using 'may contain' labelling to inform food choice: a qualitative study of nut allergic consumers. BMC Public Health. 2011;11:734.

25. Zurzolo GA, Koplin JJ, Mathai ML, Tang MK, Allen KJ. Perceptions of precautionary labelling among parents of children with food allergy and anaphylaxis. Med J Aust. 2013;198(11):621-3.

26. Jansson SA, Heibert-Arnlind M, Middelveld RJ, Bengtsson UJ, Sundqvist AC, Kallström-Bengtsson I, et al. Health-related quality of life, assessed with a disease-specific questionnaire, in Swedish adults suffering from well-diagnosed food allergy to staple foods. Clin Transl Allergy. 2013;3(1):21.

27. Protudjer JLP, Jansson SA, Östblom E, Heibert Arnlind M, Bengtsson U, Dahlen S-E, et al. Health-related quality of life in children with objectivelydiagnosed staple food allergy assessed with a disease-specific questionnaire. Acta Paediatr. 2015;104(10):1047-54.

28. Stjerna ML. Food, risk and place: agency and negotiations of young people with food allergy. Soc Health Illness. 2015;37(2):284-97.

29. Protudjer JLP, Dumontet J, McGavock JM. My Voice: a grounded theory analysis of the lived experience of type 2 diabetes in adolescence. Can J Diabetes. 2014;38(4):229-36.

30. Monks H, Gowland MH, MacKenzie H, Erlewyn-Lajeunesse M, King R, Lucas JS, et al. How do teenagers manage their food allergies? Clin Exp Allergy. 2010;40(10):1533-40.

31. Patel DA, Holdford DA, Edwards E, Carroll NV. Estimating the economic burden of food-induced allergic reactions and anaphylaxis in the United States. J Allergy Clin Immunol. 2011;128(1):110-5.

32. Protudjer JLP, Jansson SA, Arnlind Heibert M, Bengtsson U, KallströmBengtsson I, Marklund B, et al. Household costs associated with objectively-diagnosed allergy to staple foods in children and adolescents. Jllergy Clin Immunol. 2015;3(1):68-75.

33. Kivistö J, Protudjer JLP, Karjalainen J, Wickman M, Bergström A, Mattila V. Hospitalizations due to allergic reactions in Finnish and Swedish children between 1999 and 2011. Allergy. 2016;71(5):677-83.

34. Seid M, Varni JW, Segall D, Kurtin PS. Health-related quality of life as a predictor of pediatric healthcare costs: a two-year prospective cohort analysis. Health Qual Life Outcomes. 2004;2:48.

\section{Submit your next manuscript to BioMed Central and we will help you at every step:}

- We accept pre-submission inquiries

- Our selector tool helps you to find the most relevant journal

- We provide round the clock customer support

- Convenient online submission

- Thorough peer review

- Inclusion in PubMed and all major indexing services

- Maximum visibility for your research

Submit your manuscript at www.biomedcentral.com/submit
BioMed Central 